\title{
Characterization of equality in the correlation inequality for convex functions, the U-conjecture
}

\author{
Gilles Hargé \\ Equipe d'analyse et de probabilités, université d'Evry, rue du père Jarlan, bât. Maupertuis, 91025 Evry Cedex, France
}

Received 16 June 2003; received in revised form 28 May 2004; accepted 14 June 2004

Available online 22 September 2004

\begin{abstract}
We give a characterization of the equality in Hu's inequality (which is a correlation inequality between two convex functions in $\mathbb{R}^{n}$ with respect to the standard Gaussian measure). For this, we prove a new inequality which is slightly better than Hu's inequality. Then, we obtain a result concerning the U-conjecture.

๑) 2004 Elsevier SAS. All rights reserved.
\end{abstract}

\section{Résumé}

On donne une caractérisation du cas d'égalité dans l'inégalité de Hu, qui est une inégalité de décorrélation entre deux fonctions convexes contre la mesure gaussienne dans $\mathbb{R}^{n}$. Pour cela, on démontre une inégalité légèrement plus forte que l'inégalité de $\mathrm{Hu}$. On obtient aussi un résultat concernant la conjecture $\mathrm{U}$.

๑) 2004 Elsevier SAS. All rights reserved.

MSC: primary: 52A40, 60E15

Keywords: Gaussian measure; Ornstein-Uhlenbeck semigroup; Convexity; Correlation

\section{Introduction}

We denote by $\mu_{n}$ the standard Gaussian measure on $\mathbb{R}^{n}$. In 1973, Kagan, Linnik and Rao [11] considered the following question: if $P$ and $Q$ are two polynomials on $\mathbb{R}^{n}$ independent with respect to $\mu_{n}$ (such that if $X$ is a random vector on $\mathbb{R}^{n}$ of law $\mu_{n}$, then $P(X)$ and $Q(X)$ are independent random variables), is it possible to find an orthogonal transformation $U$ on $\mathbb{R}^{n}$ and an integer $k$ such that $P \circ U$ is a function of $\left(x_{1}, \ldots, x_{k}\right)$ and $Q \circ U$ is a function of $\left(x_{k+1}, \ldots, x_{n}\right)$ ? If the answer is positive, we say that $P$ and $Q$ are unlinked. This question is known as the U-conjecture. Kagan, Linnik and Rao gave a partial answer in [11]. Recently, Bhandari and Basu [5]

E-mail address: gharge@ maths.univ-evry.fr (G. Hargé). 
have shown that if $P$ and $Q$ are two convex, positive polynomials independent with respect to $\mu_{n}$ and if $P(0)=0$ then $P$ and $Q$ are unlinked. Related to this problem, Bhandari and DasGupta [6] proved in 1994 that two convex and even functions $f$ and $g$ are unlinked if they are uncorrelated (that is if $\int f g d \mu_{n}=\int f d \mu_{n} \int g d \mu_{n}$ ) under an additional hypothesis. This hypothesis is related to the Gaussian correlation conjecture which is still a conjecture in dimension greater than two (see [7] for further details and references on this conjecture), so, the result of Bhandari and DasGupta is not proved in the general case, that is, without this additional hypothesis. Recall now an inequality due to $\mathrm{Hu}$ [10] and which concerns two convex functions $f$ and $g$ in $L^{2}\left(\mu_{n}\right)$ (see [9] or [8] for the second term in the inequality):

$$
\int f g d \mu_{n} \geqslant \int f d \mu_{n} \int g d \mu_{n}+\left\langle\int x f d \mu_{n}, \int x g d \mu_{n}\right\rangle .
$$

If $\left\langle\int x f d \mu_{n}, \int x g d \mu_{n}\right\rangle \geqslant 0$, this is a correlation inequality between $f$ and $g(\langle\rangle$ is the usual scalar product on $\left.\mathbb{R}^{n}\right)$.

We will prove the following theorem, which shows in particular the result of Bhandari and DasGupta in the general case, and which gives a partial answer to the U-conjecture:

Theorem 1.1. Let $f, g: \mathbb{R}^{n} \rightarrow \mathbb{R}$ be convex functions in $L^{2}\left(\mu_{n}\right)$ for which equality holds in (1.1). Then, there exit an orthogonal transformation $U$ on $\mathbb{R}^{n}$, two vectors $\alpha_{1}$ and $\alpha_{2}$ in $\mathbb{R}^{n}$, an integer $k \in\{0, \ldots, n\}$ and two convex functions $\bar{f}: \mathbb{R}^{k} \rightarrow \mathbb{R}$ in $L^{2}\left(\mu_{k}\right)$ and $\bar{g}: \mathbb{R}^{n-k} \rightarrow \mathbb{R}$ in $L^{2}\left(\mu_{n-k}\right)$ such that, for all $x$ in $\mathbb{R}^{n}$ :

$$
f(U x)=\left\langle\alpha_{1}, x\right\rangle+\bar{f}\left(x_{1}, \ldots, x_{k}\right) \quad \text { and } g(U x)=\left\langle\alpha_{2}, x\right\rangle+\bar{g}\left(x_{k+1}, \ldots, x_{n}\right) \quad\left(x=\left(x_{1}, \ldots, x_{n}\right)\right) .
$$

Furthermore $\left(\alpha_{1}\right)_{i}=0$ if $i \leqslant k$ and $\left(\alpha_{2}\right)_{i}=0$ if $i>k$ (if $k=0$ or $k=n$, we make obvious conventions).

Of course, the condition is sufficient. Actually, it is easy to see that if $\varphi: \mathbb{R}^{n} \rightarrow \mathbb{R}$ is a function in $L^{2}\left(\mu_{n}\right)$ and if $\alpha$ belongs to $\mathbb{R}^{n}$ then $\varphi$ and the function: $x \mapsto\langle\alpha, x\rangle$ satisfy (1.1) with equality.

Secondly, we will show a generalization of the result of Bhandari and Basu:

Theorem 1.2. Denote by $X$ a random vector of law $\mu_{n}$. Let $f, g: \mathbb{R}^{n} \rightarrow \mathbb{R}$ be convex functions in $L^{2}\left(\mu_{n}\right)$. We assume that $f$ is an analytic function which verify, for all $x$ in $\mathbb{R}^{n}, f(x) \geqslant f(0)$. If $f(X)$ and $g(X)$ are independent random variables then $f$ and $g$ are unlinked.

The proof of Theorem 1.1 uses a new proof of Hu's inequality and the Ornstein-Uhlenbeck semigroup. In fact, we will prove a new inequality which is slightly better than Hu's inequality.

\section{Reinforcement of Hu's inequality}

Two functions $f$ and $g$ for which equality holds in (1.1) are not necessarily regular functions. Of course, it is possible to approximate such functions with regular functions but those last functions do not satisfy automatically (1.1) with equality. Nevertheless, that will be the case if we approximate $f$ and $g$ with the help of the Ornstein-Uhlenbeck semigroup. For this, we will show an inequality which is a reinforcement of Hu's inequality (Theorem 2.1 below) and which concerns the Ornstein-Uhlenbeck semigroup.

For the last thirty years, semigroups have been used to prove various inequalities as correlation, concentration, Poincaré and log-Sobolev inequalities. Concerning correlation inequalities, it is possible to refer to the works of Pitt [17] (perhaps the first use of the Ornstein-Uhlenbeck semigroup to prove a correlation inequality), Bakry and Michel [3], Hu [10], or my former works [7,8]. There is also related work of Houdré, Pérez-Abreu and Surgailis [9]. Concerning concentration, Poincaré and log-Sobolev inequalities, see for example the surveys of Bakry and 
Ledoux [1,2,12-14]. Furthermore, we will compare the following result with a work of Beckner [4] concerning Poincaré inequality.

We define the Ornstein-Uhlenbeck semigroup with the Mehler formula:

$$
\forall f \in L^{2}\left(\mu_{n}\right), \quad P_{t} f(x)=\int f\left(e^{-t} x+\sqrt{1-e^{-2 t}} y\right) d \mu_{n}(y) .
$$

The Ornstein-Uhlenbeck operator $L$ is defined by:

$$
\begin{aligned}
& D(L)=\left\{f \in L^{2}\left(\mu_{n}\right), \frac{P_{t} f-f}{t} \text { possesses a limit in } L^{2}\left(\mu_{n}\right) \text { when } t \text { goes to } 0\right\}, \\
& L f=\lim _{t \rightarrow 0} \frac{P_{t} f-f}{t} \text { for } f \in D(L) .
\end{aligned}
$$

It is well known that $D(L)=\left\{f \in L^{2}(\mu), \Delta f-\langle x, \nabla f\rangle \in L^{2}(\mu)\right\}$ and $L f=\Delta f-\langle x, \nabla f\rangle$ (where $\Delta f-\langle x, \nabla f\rangle$ is taken in distribution sense). Furthermore, we have:

$$
\forall f \in D(L), \forall t>0, \quad P_{t} f \in D(L) \quad \text { and } \quad \frac{d}{d t} P_{t}(f)=L P_{t} f=P_{t} L f \quad\left(\text { in } L^{2}\left(\mu_{n}\right)\right) .
$$

The properties of $P_{t}$ and $L$ we will use in the next theorems and remarks could be find in [15] or [16].

Theorem 2.1. Let $f, g: \mathbb{R}^{n} \rightarrow \mathbb{R}$ be convex functions such that $f \in L^{2}\left(\mu_{n}\right)$ and $g \in L^{2}\left(\mu_{n}\right)$, then, for all $t \geqslant 0$ :

$$
\int f g d \mu_{n} \geqslant \int f P_{t} g d \mu_{n}+\left(1-e^{-t}\right)\left\langle\int x f d \mu_{n}, \int x g d \mu_{n}\right\rangle .
$$

Remark 1. The inequality obtained in this theorem can be compared to a generalized Poincaré inequality due to Beckner [4]. He showed, for $f$ in $L^{2}\left(\mu_{n}\right)$ :

$$
\int f^{2} d \mu_{n}-\int\left(P_{t} f\right)^{2} d \mu_{n} \leqslant\left(1-e^{-2 t}\right) \int\|\nabla f\|^{2} d \mu_{n}
$$

If we choose $f=g$ in Theorem 2.1 and if we replace $t$ by $2 t$, we obtain, for a convex function $f$ (using the fact that $P_{t}$ is a symmetric semigroup with respect to $\mu_{n}$ ):

$$
\left(1-e^{-2 t}\right)\left\|\int \nabla f d \mu_{n}\right\|^{2} \leqslant \int f^{2} d \mu_{n}-\int\left(P_{t} f\right)^{2} d \mu_{n} .
$$

Remark 2. We deduce from Theorem 2.1, that if $f$ and $g$ are in $L^{2}\left(\mu_{n}\right)$ and convex, then:

$$
\int f \frac{g-P_{t} g}{t} d \mu_{n} \geqslant \frac{1-e^{-t}}{t}\left\langle\int x f d \mu_{n}, \int x g d \mu_{n}\right\rangle \text {. }
$$

So, we obtain, when $t$ goes to 0 and if $g \in D(L)$ :

$$
-\int f L g d \mu_{n} \geqslant\left\langle\int x f d \mu_{n}, \int x g d \mu_{n}\right\rangle \text {. }
$$

We will prove this inequality before Theorem 2.1 .

Remark 3. Recall that, for $g \in L^{2}\left(\mu_{n}\right), \lim _{t \rightarrow+\infty} P_{t} g=\int g d \mu_{n}$ (in $L^{2}\left(\mu_{n}\right)$ ). Consequently, if we let $t$ goes to infinity in Theorem 2.1, we recover inequality (1.1). 
Remark 4. In fact, the inequality of Theorem 2.1 is more accurate than inequality (1.1). Actually, Theorem 2.1 gives:

$$
\int f g d \mu_{n} \geqslant \int f P_{t} g d \mu_{n}+\left(1-e^{-t}\right)\left\langle\int x f d \mu_{n}, \int x g d \mu_{n}\right\rangle
$$

Furthermore, if we apply inequality (1.1) to $f$ and $P_{t} g$ (which is convex with the help of Mehler formula), we obtain:

$$
\begin{aligned}
\int f P_{t} g d \mu_{n} & \geqslant \int f d \mu_{n} \int P_{t} g d \mu_{n}+\left\langle\int x f d \mu_{n}, \int x P_{t} g d \mu_{n}\right\rangle \\
& \geqslant \int f d \mu_{n} \int g d \mu_{n}+e^{-t}\left\langle\int x f d \mu_{n}, \int x g d \mu_{n}\right\rangle,
\end{aligned}
$$

because $\int x P_{t} g d \mu_{n}=\int g P_{t} x d \mu_{n}=e^{-t} \int x g d \mu_{n}$. Consequently:

$$
\begin{aligned}
\int f g d \mu_{n} & \geqslant \int f P_{t} g d \mu_{n}+\left(1-e^{-t}\right)\left\langle\int x f d \mu_{n}, \int x g d \mu_{n}\right\rangle \\
& \geqslant \int f d \mu_{n} \int g d \mu_{n}+\left\langle\int x f d \mu_{n}, \int x g d \mu_{n}\right\rangle .
\end{aligned}
$$

Remark 5. Theorem 2.1 allows us to show that if $f$ and $g$ are convex functions and satisfy (1.1) with equality then $P_{t} f$ and $P_{t} g$ verify the same equality. Actually, we have:

$$
\begin{aligned}
\int f g d \mu_{n} & \geqslant \int f P_{2 t} g d \mu_{n}+\left(1-e^{-2 t}\right)\left\langle\int x f d \mu_{n}, \int x g d \mu_{n}\right\rangle \\
& \geqslant \int f d \mu_{n} \int g d \mu_{n}+\left\langle\int x f d \mu_{n}, \int x g d \mu_{n}\right\rangle
\end{aligned}
$$

If $f$ and $g$ satisfy (1.1) with equality then:

$$
\begin{aligned}
& \int f P_{2 t} g d \mu_{n}=\int f d \mu_{n} \int g d \mu_{n}+e^{-2 t}\left\langle\int x f d \mu_{n}, \int x g d \mu_{n}\right\rangle \\
& \Rightarrow \int P_{t} f P_{t} g d \mu_{n}=\int P_{t} f d \mu_{n} \int P_{t} g d \mu_{n}+\left\langle\int x P_{t} f d \mu_{n}, \int x P_{t} g d \mu_{n}\right\rangle .
\end{aligned}
$$

Before given the proof of Theorem 2.1, we will show the following result:

Theorem 2.2. If $f, g: \mathbb{R}^{n} \rightarrow \mathbb{R}$ are convex functions in $L^{2}\left(\mu_{n}\right)$ and if $g \in D(L)$, then:

$$
-\int f L g d \mu_{n} \geqslant\left\langle\int x f d \mu_{n}, \int x g d \mu_{n}\right\rangle \text {. }
$$

Proof. We will prove this theorem for $P_{u} f$ and $P_{u} g(u>0)$ instead of $f$ and $g$. Then, we will deduce easily the result for $f$ and $g$ with the following convergences in $L^{2}\left(\mu_{n}\right)$ :

$$
\lim _{u \rightarrow 0} P_{u} f=f, \quad \lim _{u \rightarrow 0} P_{u} g=g, \quad \lim _{u \rightarrow 0} L P_{u} g=\lim _{u \rightarrow 0} P_{u} L g=L g .
$$

We will use the following properties of $P_{t}$ and $L$.

- $\forall h \in L^{2}\left(\mu_{n}\right), \forall t>0, x \mapsto P_{t} h(x)$ is a $C^{\infty}$ function on $\mathbb{R}^{n}, \frac{\partial}{\partial x_{i}}\left(P_{t} h\right) \in L^{2}\left(\mu_{n}\right)$ and $\forall x \in \mathbb{R}^{n}, \forall s \geqslant 0$, $\frac{\partial}{\partial x_{i}}\left(P_{s+t} h\right)(x)=e^{-t} P_{S}\left(\frac{\partial}{\partial x_{i}}\left(P_{t} h\right)\right)(x)$. 
- $\forall\left(h_{1}, h_{2}\right) \in D(L)^{2}, \int h_{1} L h_{2} d \mu_{n}=-\int\left\langle\nabla h_{1}, \nabla h_{2}\right\rangle d \mu_{n}$.

We notice that $P_{u} f$ and $P_{u} g$ are convex, $C^{\infty}$ and in $D(L)$.

Define $\theta(t)=\int\left\langle\nabla P_{u} f, P_{t} \nabla P_{u} g\right\rangle d \mu_{n}$. Because $u$ is strictly positive, $\nabla P_{u} f$ and $\nabla P_{u} g$ are in $L^{2}\left(\mu_{n}\right)$. Consequently:

$$
\lim _{t \rightarrow \infty} \theta(t)=\left\langle\int \nabla P_{u} f d \mu_{n}, \int \nabla P_{u} g d \mu_{n}\right\rangle
$$

Moreover:

$$
\theta^{\prime}(t)=\sum_{i=1}^{n} \int \frac{\partial P_{u} f}{\partial x_{i}} L P_{t}\left(\frac{\partial P_{u} g}{\partial x_{i}}\right) d \mu_{n}=-\sum_{i=1}^{n} \int\left\langle\nabla\left[\frac{\partial P_{u} f}{\partial x_{i}}\right], \nabla\left[P_{t}\left(\frac{\partial P_{u} g}{\partial x_{i}}\right)\right]\right\rangle d \mu_{n} .
$$

It is possible to justify this equality by saying that $P_{t}\left(\frac{\partial P_{u} g}{\partial x_{i}}\right)$ belongs to $D(L)$ and that $\frac{\partial P_{u} f}{\partial x_{i}}$ belongs to $D(L)$ (because $\left.\frac{\partial P_{u} f}{\partial x_{i}}=e^{-u / 2} P_{u / 2}\left(\frac{\partial}{\partial x_{i}}\left(P_{u / 2} f\right)\right)\right)$. We write:

$$
P_{t}\left(\frac{\partial P_{u} g}{\partial x_{i}}\right)=e^{t} \frac{\partial}{\partial x_{i}} P_{t} P_{u} g
$$

then, we obtain:

$$
\theta^{\prime}(t)=-e^{t} \sum_{i=1}^{n} \int\left\langle\nabla\left[\frac{\partial P_{u} f}{\partial x_{i}}\right], \nabla\left[\frac{\partial P_{t+u} g}{\partial x_{i}}\right]\right\rangle d \mu_{n}=-e^{t} \int \operatorname{Tr}\left(\operatorname{Hess} P_{u} f \text { Hess } P_{t+u} g\right) d \mu_{n} .
$$

Because $P_{u} f$ and $P_{t+u} g$ are convex, we deduce $\theta^{\prime}(t) \leqslant 0$. Consequently:

$$
\int\left\langle\nabla P_{u} f, \nabla P_{u} g\right\rangle d \mu_{n} \geqslant\left\langle\int \nabla P_{u} f d \mu_{n}, \int \nabla P_{u} g d \mu_{n}\right\rangle,
$$

which gives:

$$
-\int P_{u} f L P_{u} g d \mu_{n} \geqslant\left\langle\int x P_{u} f d \mu_{n}, \int x P_{u} g d \mu_{n}\right\rangle .
$$

Proof of Theorem 2.1. For two convex functions $f$ and $g$ in $L^{2}\left(\mu_{n}\right)$, we define:

$$
\begin{aligned}
\xi(t) & =\int f P_{t} g d \mu_{n}-\left\langle\int x f d \mu_{n}, \int x P_{t} g d \mu_{n}\right\rangle \\
& =\int f P_{t} g d \mu_{n}-e^{-t}\left\langle\int x f d \mu_{n}, \int x g d \mu_{n}\right\rangle .
\end{aligned}
$$

It is sufficient to show that $\xi$ is a decreasing function.

$$
\begin{aligned}
\xi^{\prime}(t) & =\int f L P_{t} g d \mu_{n}-\left\langle\int x f d \mu_{n}, \int x L P_{t} g d \mu_{n}\right\rangle \\
& =\int f L P_{t} g d \mu_{n}-\left\langle\int x f d \mu_{n}, \int L x P_{t} g d \mu_{n}\right\rangle \\
& =\int f L P_{t} g d \mu_{n}+\left\langle\int x f d \mu_{n}, \int x P_{t} g d \mu_{n}\right\rangle .
\end{aligned}
$$

We apply the previous theorem to $f$ and $P_{t} g$ to obtain $\xi^{\prime}(t) \leqslant 0$. 


\section{Characterization of equality in Hu's inequality}

We begin to prove some elementary facts concerning convex functions. The following reasoning process is inspired by the one of Bhandari and DasGupta [6].

Lemma 3.1. If $\varphi: \mathbb{R} \rightarrow \mathbb{R}$ is a convex and non-constant function then $\lim _{t \rightarrow+\infty} \varphi(t)=+\infty$ or $\lim _{t \rightarrow-\infty} \varphi(t)=$ $+\infty[6]$.

We deduce, by using the convexity of $\varphi(x)-\langle\alpha, x\rangle$ :

If $\varphi: \mathbb{R}^{n} \rightarrow \mathbb{R}$ is convex and verify

$\exists \alpha \in \mathbb{R}^{n}, \exists b \in \mathbb{R}, \forall x \in \mathbb{R}^{n}, \quad \varphi(x) \leqslant\langle\alpha, x\rangle+b$,

then: $\forall x \in \mathbb{R}^{n}, \varphi(x)=\langle\alpha, x\rangle+\varphi(0)$.

Lemma 3.2. Let $f: \mathbb{R}^{n} \rightarrow \mathbb{R}$ be a convex function. We define:

$$
\mathcal{E}(f)=\left\{h \in \mathbb{R}^{n}, \exists(a, b) \in \mathbb{R}^{2}, \forall t \in \mathbb{R}, f(t h) \leqslant a t+b\right\} .
$$

$\mathcal{E}(f)$ is a linear space contained in $\mathbb{R}^{n}$ and

$$
\exists ! \alpha \in \mathcal{E}(f), \forall y_{1} \in \mathcal{E}(f), \forall y_{2} \in \mathbb{R}^{n}, \quad f\left(y_{1}+y_{2}\right)=\left\langle\alpha, y_{1}\right\rangle+f\left(y_{2}\right) .
$$

Proof. It is obvious to see that $\mathcal{E}(f)$ is a linear space. Let $\left(e_{1}, \ldots e_{r}\right)$ be an orthonormal basis of $\mathcal{E}(f)$ and $y$ be an element of $\mathbb{R}^{n}$. We denote $a_{i}$ and $b_{i}$ the numbers associated to $e_{i}$ in the definition of $\mathcal{E}(f)$. We have:

$$
\begin{aligned}
f\left(\sum_{i=1}^{r} x_{i} e_{i}+y\right) & \leqslant \frac{1}{r+1}\left(\sum_{i=1}^{r} f\left((r+1) x_{i} e_{i}\right)+f((r+1) y)\right) \\
& \leqslant\left\langle\alpha, \sum_{i=1}^{r} x_{i} e_{i}\right\rangle+\frac{1}{r+1}\left(\sum_{i=1}^{r} b_{i}+f((r+1) y)\right),
\end{aligned}
$$

where $\alpha=\sum_{i=1}^{r} a_{i} e_{i}$. For a fixed $y$, the map: $\left(x_{1}, \ldots x_{r}\right) \mapsto f\left(\sum_{i=1}^{r} x_{i} e_{i}+y\right)$ is convex, so, we know from Lemma 3.1 that:

$$
\forall\left(x_{1}, \ldots, x_{r}\right) \in \mathbb{R}^{r}, \quad f\left(\sum_{i=1}^{r} x_{i} e_{i}+y\right)=\left\langle\alpha, \sum_{i=1}^{r} x_{i} e_{i}\right\rangle+f(y) .
$$

Unicity of $\alpha$ is obvious.

Remark 6. If we choose $y_{2}=0$ and $h \in \mathcal{E}(f)$ we see that $\forall t \in \mathbb{R}, f(t h)=t\langle\alpha, h\rangle+f(0)$.

Lemma 3.3. Let $\varphi: \mathbb{R}^{n} \rightarrow \mathbb{R}$ be a convex function in $L^{2}\left(\mu_{n}\right)$. Let $h \in \mathbb{R}^{n}$, we assume that the map: $t \mapsto \varphi(t h)$ is a non-constant function. Let $\left(e_{1}, \ldots, e_{k}\right)$ be an orthonormal family in $\mathbb{R}^{n}(k \leqslant n)$ and define:

$$
\tilde{\varphi}(x)=\int \varphi\left(x+\sum_{i=1}^{k} y_{i} e_{i}\right) d \mu_{k}(y) \quad \text { where } y=\left(y_{1}, \ldots, y_{k}\right) .
$$

Then:

$$
\begin{aligned}
& \exists \varepsilon \in\{-1,1\}, \quad \lim _{\varepsilon t \rightarrow+\infty} \tilde{\varphi}(t h)=+\infty, \\
& \exists \varepsilon \in\{-1,1\}, \quad \forall s \geqslant 0, \quad \lim _{\varepsilon t \rightarrow+\infty}\left(P_{s} \varphi\right)(t h)=+\infty .
\end{aligned}
$$


Proof. We know from Lemma 3.1 that

$$
\exists \varepsilon \in\{-1,1\}, \quad \lim _{\varepsilon t \rightarrow+\infty} \varphi(t h)=+\infty .
$$

Let $y \in \mathbb{R}^{n}: \varphi\left(\frac{t}{2} h\right) \leqslant \frac{1}{2}(\varphi(t h+y)+\varphi(-y))$ so $\lim _{\varepsilon t \rightarrow+\infty} \varphi(t h+y)=+\infty$. Furthermore:

$$
\exists A>0, \varepsilon t>A \Rightarrow \varphi\left(\frac{t}{2} h\right) \geqslant 0 .
$$

With Fatou's lemma, we write:

$$
\begin{aligned}
& \int\left(\liminf _{\varepsilon t \rightarrow+\infty}\left[\varphi\left(t h+\sum_{i=1}^{k} y_{i} e_{i}\right)+\varphi\left(-\sum_{i=1}^{k} y_{i} e_{i}\right)\right] d \mu_{k}(y)\right) \\
& \leqslant \liminf _{\varepsilon t \rightarrow+\infty} \int\left[\varphi\left(t h+\sum_{i=1}^{k} y_{i} e_{i}\right)+\varphi\left(-\sum_{i=1}^{k} y_{i} e_{i}\right)\right] d \mu_{k}(y) \\
& \Rightarrow \lim _{\varepsilon t \rightarrow+\infty} \tilde{\varphi}(t h)=+\infty
\end{aligned}
$$

We can prove the result for $P_{s} \varphi$ in the same way.

Lemma 3.4. Let $f: \mathbb{R}^{n} \rightarrow \mathbb{R}$ be a convex function in $L^{2}(\mu)$. Let $h \in \mathbb{R}^{n}$ and $s \geqslant 0$. Like in the previous lemma, we associate $\tilde{f}$ to $f$, then:

$$
h \notin \mathcal{E}(f) \Rightarrow\left(h \notin \mathcal{E}(\tilde{f}) \text { and } h \notin \mathcal{E}\left(P_{s} f\right)\right) .
$$

Proof. $h \notin \mathcal{E}(f)$ so, for every $a$ in $\mathbb{R}$, the map $t \mapsto f(t h)-a t$ is non-constant. Define $f_{a}(x)=f(x)-a\left\langle x, \frac{h}{\|h\|^{2}}\right\rangle$. $f_{a}$ is convex and the map $t \rightarrow f_{a}(t h)$ is non-constant, so, with the previous lemma:

$$
\exists \varepsilon \in\{-1,1\}, \quad \lim _{\varepsilon t \rightarrow+\infty} \tilde{f}_{a}(t h)=+\infty .
$$

Moreover: $\int f_{a}\left(t h+\sum_{i=1}^{k} y_{i} e_{i}\right) d \mu_{k}(y)=\int f\left(t h+\sum_{i=1}^{k} y_{i} e_{i}\right) d \mu_{k}(y)-a t=\tilde{f}(t h)-a t$. We deduce that the map $t \rightarrow \tilde{f}(t h)-a t$ is non-constant for all $a$. So $h \notin \mathcal{E}(\tilde{f})$. We can prove the result for $P_{s} \varphi$ in the same way.

Remark 7. In fact, the following equalities are true: $\mathcal{E}(f)=\mathcal{E}(\tilde{f})=\mathcal{E}\left(P_{s} f\right)$. Actually, we have for all $\beta \in[0, \sqrt{2}$ [ and because $f \in L^{2}\left(\mu_{n}\right): \int|f(\beta x)| d \mu_{n}(x)<+\infty$. Let $h \in \mathcal{E}(f)$ then $\exists(a, b) \in \mathbb{R}^{2}, \forall t \in \mathbb{R}, f(t h) \leqslant a t+b$. For $y \in \mathbb{R}^{n}$ and $\left.\beta \in\right] 1, \sqrt{2}\left[\right.$, we write: $t h+y=\left(1-\frac{1}{\beta}\right) \frac{\beta}{\beta-1} t h+\frac{1}{\beta} \beta y$, consequently:

$$
f(t h+y) \leqslant a t+\left(1-\frac{1}{\beta}\right) b+\frac{1}{\beta} f(\beta y) .
$$

We deduce: $h \in \mathcal{E}(\tilde{f})$ and $h \in \mathcal{E}\left(P_{s} f\right)$.

Now, we can prove Theorem 1.1.

Proof of Theorem 1.1. We choose $\left(e_{1}, \ldots, e_{n}\right)$ an orthonormal basis of $\mathbb{R}^{n}$ such that:

- $\left(e_{1}, \ldots, e_{r+k}\right)$ is an orthonormal basis of $\mathcal{E}(f)^{\perp}$,

- $\left(e_{r+1}, \ldots, e_{r+k}\right)$ is an orthonormal basis of $\mathcal{E}(f)^{\perp} \cap \mathcal{E}(g)$. 
We use Lemma 3.2 to construct $\alpha_{1}$ associated to $f$ and $\alpha_{2}$ associated to $g$. We obtain:

$$
\begin{aligned}
f\left(\sum_{i=1}^{n} x_{i} e_{i}\right) & =f\left(\sum_{i=1}^{r+k} x_{i} e_{i}\right)+\left\langle\alpha_{1}, \sum_{i=r+k+1}^{n} x_{i} e_{i}\right\rangle, \\
g\left(\sum_{i=1}^{n} x_{i} e_{i}\right) & =g\left(\sum_{i=1}^{r} x_{i} e_{i}+\sum_{i=r+k+1}^{n} x_{i} e_{i}\right)+\left\langle\alpha_{2}, \sum_{i=r+1}^{r+k} x_{i} e_{i}\right\rangle .
\end{aligned}
$$
$\neq 0$.

We want to prove $r=0$ so we assume $r \neq 0$. We will use the fact that $\sum_{i=1}^{r} x_{i} e_{i} \notin \mathcal{E}(f) \cup \mathcal{E}(g)$ if $\left(x_{1}, \ldots, x_{r}\right)$

Define (abuse of notations):

$$
\begin{aligned}
& \tilde{f}\left(x_{1}, \ldots, x_{r}\right)=\tilde{f}\left(\sum_{i=1}^{r} x_{i} e_{i}\right)=\int f\left(\sum_{i=1}^{r+k} x_{i} e_{i}\right) d \mu_{k}\left(x_{r+1}, \ldots, x_{r+k}\right) \\
& \tilde{g}\left(x_{1}, \ldots, x_{r}\right)=\tilde{g}\left(\sum_{i=1}^{r} x_{i} e_{i}\right)=\int g\left(\sum_{i=1}^{r} x_{i} e_{i}+\sum_{i=r+k+1}^{n} x_{i} e_{i}\right) d \mu_{n-r-k}\left(x_{r+k+1}, \ldots, x_{n}\right) .
\end{aligned}
$$

Recall that if $\varphi: \mathbb{R}^{n} \rightarrow \mathbb{R}$ is a function in $L^{2}\left(\mu_{n}\right)$ and if $\alpha \in \mathbb{R}^{n}$ then $\varphi$ and the map $x \mapsto\langle\alpha, x\rangle$ satisfy (1.1) with equality. So, because $f$ and $g$ satisfy (1.1) with equality, it is the case for $\tilde{f}$ and $\tilde{g}$ against $\mu_{r}$ in $\mathbb{R}^{r}$. Furthermore (Lemma 3.4) $\sum_{i=1}^{r} x_{i} e_{i} \notin \mathcal{E}(\tilde{f}) \cup \mathcal{E}(\tilde{g})$ if $\left(x_{1}, \ldots, x_{r}\right) \neq 0$. We deduce $\mathcal{E}(\tilde{f})=\mathcal{E}(\tilde{g})=\{0\}$.

Using Remark 4 and because $\tilde{f}$ and $\tilde{g}$ satisfy (1.1) with equality, we have:

$$
\forall u \geqslant 0, \quad \int \tilde{f} \tilde{g} d \mu_{r}=\int \tilde{f} P_{u} \tilde{g} d \mu_{r}+\left(1-e^{-u}\right)\left\langle\int x \tilde{f} d \mu_{r}, \int x \tilde{g} d \mu_{r}\right\rangle,
$$

(the Ornstein-Uhlenbeck semigroup we use here is the one of $\mathbb{R}^{r}$ ).

For the continuation of the proof, we only need the existence of one real $u>0$ such that this equality is verified. The function $\xi$ used in the proof of Theorem 2.1 is decreasing, so we deduce:

$$
\forall s \in[0, u], \quad \xi^{\prime}(s)=0 .
$$

Consequently, for all $s \in[0, u]$ :

$$
\begin{aligned}
&-\int \tilde{f} L P_{s} \tilde{g} d \mu_{r}=\left\langle\int x \tilde{f} d \mu_{r}, \int x P_{s} \tilde{g} d \mu_{r}\right\rangle \\
& \Rightarrow-\int P_{S / 2} \tilde{f} L P_{S / 2} \tilde{g} d \mu_{r}=\left\langle\int x \tilde{f} d \mu_{r}, \int P_{s / 2} x P_{S / 2} \tilde{g} d \mu_{r}\right\rangle \\
&=e^{s / 2}\left\langle\int x \tilde{f} d \mu_{r}, \int x P_{s / 2} \tilde{g} d \mu_{r}\right\rangle \\
&=\left\langle\int x P_{s / 2} \tilde{f} d \mu_{r}, \int x P_{s / 2} \tilde{g} d \mu_{r}\right\rangle \\
& \Rightarrow \int\left\langle\nabla P_{S / 2} \tilde{f}, \nabla P_{s / 2} \tilde{g}\right\rangle d \mu_{r}=\left\langle\int \nabla P_{s / 2} \tilde{f} d \mu_{r}, \int \nabla P_{S / 2} \tilde{g} d \mu_{r}\right\rangle
\end{aligned}
$$

We define, like in the proof of Theorem 2.2, the function:

$$
\theta(t)=\int\left\langle\nabla P_{s / 2} \tilde{f}, P_{t} \nabla P_{s / 2} \tilde{g}\right\rangle d \mu_{r}
$$


So we have, for every $t \geqslant 0, \theta^{\prime}(t)=0$, consequently:

$$
\forall t \geqslant 0, \quad \int \operatorname{Tr}\left(\operatorname{Hess} P_{s / 2} \tilde{f} \operatorname{Hess} P_{t+s / 2} \tilde{g}\right) d \mu_{r}=0 .
$$

Using the formula Hess $P_{t+s / 2} \tilde{g}=e^{-2 t} P_{t}\left(\right.$ Hess $\left.P_{s / 2} \tilde{g}\right)$, we obtain:

$$
\forall t \geqslant 0, \quad \int \operatorname{Tr}\left(\operatorname{Hess} P_{s / 2} \tilde{f} P_{t}\left(\operatorname{Hess} P_{s / 2} \tilde{g}\right)\right) d \mu_{r}=0 .
$$

Let $t$ goes to infinity, then:

$$
\operatorname{Tr}\left(\int \operatorname{Hess} P_{s / 2} \tilde{f} d \mu_{r} \int \operatorname{Hess} P_{s / 2} \tilde{g} d \mu_{r}\right)=0 .
$$

Matrices $\int \operatorname{Hess} P_{s / 2} \tilde{f} d \mu_{r}$ and $\int \operatorname{Hess} P_{s / 2} \tilde{g} d \mu_{r}$ are symmetric and positive. It is easy to see that if both are invertible then equality (3.1) is impossible. So, we can assume that $\int \operatorname{Hess} P_{s / 2} \tilde{f} d \mu_{r}$ is not invertible. We deduce there exists an element $h$ of $\mathbb{R}^{r}, h \neq 0$, such that:

$$
\begin{aligned}
& \left\langle\left[\int \operatorname{Hess} P_{s / 2} \tilde{f} d \mu_{r}\right] h, h\right\rangle=0 \\
& \quad \Rightarrow \int\left\langle\left[\operatorname{Hess} P_{s / 2} \tilde{f}\right] h, h\right\rangle d \mu_{r}=0 \\
& \quad \Rightarrow \forall x \in \mathbb{R}^{r}, \quad\left\langle\left[\operatorname{Hess} P_{s / 2} \tilde{f}\right](x) h, h\right\rangle=0 .
\end{aligned}
$$

Define $\varsigma(\lambda)=P_{s / 2} \tilde{f}(\lambda h)$, we obtain, for all $\lambda, \varsigma^{\prime \prime}(\lambda)=0$, consequently:

$$
\exists(a, b) \in \mathbb{R}^{2}, \forall \lambda \in \mathbb{R}, \quad P_{s / 2} \tilde{f}(\lambda h)=a \lambda+b .
$$

So $h \in \mathcal{E}\left(P_{S / 2} \tilde{f}\right)$. Then, we deduce from Lemma 3.4 that $h \in \mathcal{E}(\tilde{f})$ but this is impossible because $\mathcal{E}(\tilde{f})=\{0\}$.

Remark 8. Actually, we have proved that if $f$ and $g$ are two convex functions for which equality holds in Theorem 2.1 for a fixed $t>0$, then the conclusion of Theorem 1.1 remains valid.

Remark 9. We obtain in the proof of the theorem: $\alpha_{1} \in \mathcal{E}(f)$ and $\alpha_{2} \in \mathcal{E}(g)$.

We deduce immediately from Theorem 1.1:

Corollary 3.5. Let $f, g: \mathbb{R}^{n} \rightarrow \mathbb{R}$ be two convex functions in $L^{2}\left(\mu_{n}\right)$ such that $\int f g d \mu_{n}=\int f d \mu_{n} \int g d \mu_{n}$ and $\left\langle\int x f d \mu_{n}, \int x g d \mu_{n}\right\rangle \geqslant 0$. Then $\left\langle\int x f d \mu_{n}, \int x g d \mu_{n}\right\rangle=0$ and there exist an orthogonal transformation $U$ on $\mathbb{R}^{n}$, two vectors $\alpha_{1}$ and $\alpha_{2}$ in $\mathbb{R}^{n}$, an integer $k \in\{0, \ldots, n\}$ and two convex functions $\bar{f}: \mathbb{R}^{k} \rightarrow \mathbb{R}$ in $L^{2}\left(\mu_{k}\right)$ and $\bar{g}: \mathbb{R}^{n-k} \rightarrow \mathbb{R}$ in $L^{2}\left(\mu_{n-k}\right)$ such that, for every $x$ in $\mathbb{R}^{n}:$

$$
f(U x)=\left\langle\alpha_{1}, x\right\rangle+\bar{f}\left(x_{1}, \ldots, x_{k}\right) \quad \text { and } \quad g(U x)=\left\langle\alpha_{2}, x\right\rangle+\bar{g}\left(x_{k+1}, \ldots, x_{n}\right) \quad\left(x=\left(x_{1}, \ldots, x_{n}\right)\right) .
$$

We have $\left(\alpha_{1}\right)_{i}=0$ if $i \leqslant k$ and $\left(\alpha_{2}\right)_{i}=0$ if $i>k$.

Moreover, if $\int x f d \mu_{n}=\int x g d \mu_{n}=0$ then $\left\langle\int x f d \mu_{n}, \int x g d \mu_{n}\right\rangle=0$ is verified and $\alpha_{1}=\alpha_{2}=0$ (consequently, $f$ and $g$ are unlinked).

Proof. We start with Hu's inequality:

$$
\int f g d \mu_{n} \geqslant \int f d \mu_{n} \int g d \mu_{n}+\left\langle\int x f d \mu_{n}, \int x g d \mu_{n}\right\rangle \geqslant \int f d \mu_{n} \int g d \mu_{n} .
$$


We obtain: $\left\langle\int x f d \mu_{n}, \int x g d \mu_{n}\right\rangle=0$. Then, we use Theorem 1.1 to construct $U$.

Now, assume that $\int x f d \mu_{n}=0$. Denote $x=\left(y_{1}, y_{2}\right)$ where $y_{1}=\left(x_{1}, \ldots, x_{k}\right)$ and $y_{2}=\left(x_{k+1}, \ldots, x_{n}\right), \alpha_{1}=$ $(0, \bar{\alpha})$ with $\bar{\alpha} \in \mathbb{R}^{n-k}$. We obtain: $\int x f(U x) d \mu_{n}(x)=\left(\int y_{1} \bar{f}\left(y_{1}\right) d \mu_{k}\left(y_{1}\right), \bar{\alpha}\right)$ and $\bar{\alpha}=0$.

Remark 10. The second part of this corollary generalizes and proves the result of Bhandari and DasGupta [6] in any dimension (if $f$ and $g$ are even functions then $\int x f d \mu_{n}=\int x g d \mu_{n}=0$ ).

\section{The U-conjecture}

In the following, we denote by $X=\left(X_{1}, \ldots, X_{n}\right)$ a random vector of law $\mu_{n}$. We deduce from the previous corollary:

Theorem 4.1. Let $f, g: \mathbb{R}^{n} \rightarrow \mathbb{R}$ be two convex functions in $L^{2}\left(\mu_{n}\right)$. Assume that $\int x f d \mu_{n}=0$ and that $f(X)$ and $g(X)$ are independent random variables then $f$ and $g$ are unlinked.

Proof. The equality $\left\langle\int x f d \mu_{n}, \int x g d \mu_{n}\right\rangle=0$ is verified. With notations and results of the first part of the previous corollary, we obtain $\alpha_{1}=0$. So, it is possible to find $U$ and $\bar{f}$ such that:

$$
f(U x)=\bar{f}\left(x_{1}, \ldots, x_{k}\right) \text { and } g(U x)=a x_{1}+\bar{g}\left(x_{k+1}, \ldots, x_{n}\right) .
$$

It is easy to see that $f(U X)$ and $g(U X)$ are independent random variables. Denote $Y=\left(X_{2}, \ldots, X_{k}\right)$ and $Z=$ $\left(X_{k+1}, \ldots, X_{n}\right) \cdot \bar{f}\left(X_{1}, Y\right)$ and $a X_{1}+\bar{g}(Z)$ are independent random variables, so it is the case for $\bar{f}\left(X_{1}, Y\right)$ and $a X_{1}$. Recall that in the proof of Theorem 1.1, we have obtained:

$$
\bar{f}\left(x_{r+1}, \ldots, x_{r+k}\right)=f\left(\sum_{i=r+1}^{r+k} x_{i} e_{i}\right),
$$

where, for $r+1 \leqslant i \leqslant r+k, e_{i} \in \mathcal{E}(f)^{\perp}$. Consequently, $\sum_{i=r+1}^{r+k} x_{i} e_{i} \notin \mathcal{E}(f)$ if $\left(x_{r+1}, \ldots, x_{r+k}\right) \neq 0$. So, we have $\mathcal{E}(\bar{f})=\{0\}$. Let $\psi \in L^{2}\left(\mu_{1}\right)$ and let us assume $a \neq 0$ and $\bar{f}$ depends on $x_{1}$.

$$
E\left(\bar{f}\left(X_{1}, Y\right) \psi\left(a X_{1}\right)\right)=E\left(\bar{f}\left(X_{1}, Y\right)\right) E\left(\psi\left(a X_{1}\right)\right)=E\left(\int \bar{f}\left(X_{1}, y\right) d \mu_{k-1}(y) \psi\left(a X_{1}\right)\right) .
$$

Define: $\tilde{f}\left(x_{1}\right)=\int \bar{f}\left(x_{1}, y\right) d \mu_{k-1}(y)$. We have $\mathcal{E}(\tilde{f})=\{0\}$ (Lemma 3.4). Furthermore:

$$
E\left(\tilde{f}\left(X_{1}\right) \psi\left(a X_{1}\right)\right)=E\left(\tilde{f}\left(X_{1}\right)\right) E\left(\psi\left(a X_{1}\right)\right) .
$$

We choose $\psi\left(x_{1}\right)=\tilde{f}\left(\frac{1}{a} x_{1}\right)$. We obtain: $\tilde{f}\left(X_{1}\right)=E\left(\tilde{f}\left(X_{1}\right)\right)$ almost surely. So, $\tilde{f}$ is a constant function, which is a contradiction with $\mathcal{E}(\tilde{f})=\{0\}$. We deduce $a=0$ or $\bar{f}$ does not depend on $x_{1}$.

The following result is to be compared to the one of Bhandari and Basu [5] who show that if $P$ and $Q$ are two convex, positive polynomials independent with respect to $\mu$ and if $P(0)=0$ then $P$ and $Q$ are unlinked.

Corollary 4.2. Let $f, g: \mathbb{R}^{n} \rightarrow \mathbb{R}$ be two convex functions in $L^{2}\left(\mu_{n}\right)$. We assume that $f$ and $g$ are bounded below, that $\left\langle\int x f d \mu_{n}, \int x g d \mu_{n}\right\rangle=0$ and that $\int f g d \mu_{n}=\int f d \mu_{n} \int g d \mu_{n}$ then $f$ and $g$ are unlinked.

Proof. We use notations and results of the first part of Corollary 3.5. Because $f$ and $g$ are bounded below, it is easy to see that $\alpha_{1}=\alpha_{2}=0$.

Now, we will prove Theorem 1.2. We begin with a lemma: 
Lemma 4.3. Let $f: \mathbb{R}^{n} \rightarrow \mathbb{R}$ be a convex function such that:

$$
\forall x \in \mathbb{R}^{n}, x \neq 0 \Rightarrow f(x)>f(0) .
$$

For $\varepsilon>0$, define $\delta(\varepsilon)=\sup \left\{\|x\|, x \in \mathbb{R}^{n}, f(x)-f(0) \leqslant \varepsilon\right\}$ then $\lim _{\varepsilon \rightarrow 0} \delta(\varepsilon)=0$.

Proof. Assume that $\delta(\varepsilon)$ does not go to 0 . We construct $\eta>0$ and a sequence $\left(\varepsilon_{p}\right)_{p} \geqslant 1$ such that $\lim _{p \rightarrow+\infty} \varepsilon_{p}=0$ and $\delta\left(\varepsilon_{p}\right)>\eta$ for all $p$. We associate to $\varepsilon_{p}$ an element $x_{p}$ of $\mathbb{R}^{n}$ such that $\left\|x_{p}\right\|>\eta$ and $f\left(x_{p}\right)-f(0) \leqslant \varepsilon_{p}$. We can assume that $\frac{x_{p}}{\left\|x_{p}\right\|}$ goes to $e$ (an element of $\mathbb{R}^{n}$ of norm equal to 1 ). Then:

$$
f\left(\frac{\eta}{\left\|x_{p}\right\|} x_{p}\right) \leqslant\left(1-\frac{\eta}{\left\|x_{p}\right\|}\right) f(0)+\frac{\eta}{\left\|x_{p}\right\|} f\left(x_{p}\right) \leqslant f(0)+\varepsilon_{p} .
$$

We obtain: $f(\eta e) \leqslant f(0)$, so $f(\eta e)=f(0)$, but it is not possible because $\eta e \neq 0$.

Proof of Theorem 1.2. We use here the same orthonormal basis as in the proof of Theorem 1.1. So, we construct $\alpha_{1}$ and $\alpha_{2}$ associated to $f$ and $g$. Because $f$ is bounded below, we have $\alpha_{1}=0$. Consequently:

$$
\begin{aligned}
f\left(\sum_{i=1}^{n} x_{i} e_{i}\right) & =f\left(\sum_{i=1}^{r+k} x_{i} e_{i}\right) \\
g\left(\sum_{i=1}^{n} x_{i} e_{i}\right) & =g\left(\sum_{i=1}^{r} x_{i} e_{i}+\sum_{i=r+k+1}^{n} x_{i} e_{i}\right)+\left\langle\alpha_{2}, \sum_{i=r+1}^{r+k} x_{i} e_{i}\right\rangle .
\end{aligned}
$$

We assume $r \geqslant 1$. Define:

$$
\begin{aligned}
& \tilde{g}\left(x_{1}, \ldots, x_{r}\right)=\int g\left(\sum_{i=1}^{r} x_{i} e_{i}+\sum_{i=r+k+1}^{n} x_{i} e_{i}\right) d \mu_{n-(r+k)}\left(x_{r+k+1}, \ldots, x_{n}\right), \\
& f_{1}\left(x_{1}, \ldots, x_{r+k}\right)=f\left(\sum_{i=1}^{r+k} x_{i} e_{i}\right) .
\end{aligned}
$$

We have $\mathcal{E}(\tilde{g})=\{0\}$ (Lemma 3.4) and $\mathcal{E}\left(f_{1}\right)=\{0\}$ (because $\left(e_{1}, \ldots, e_{r+k}\right)$ is an orthonormal basis of $\mathcal{E}(f)^{\perp}$ ). Let $\psi: \mathbb{R} \rightarrow \mathbb{R}$ be measurable and bounded, we obtain:

$$
\begin{aligned}
& \int\left(\tilde{g}\left(x_{1}, \ldots, x_{r}\right)+\left\langle\alpha_{2}, \sum_{i=r+1}^{r+k} x_{i} e_{i}\right\rangle\right) \psi\left(f_{1}\right)\left(x_{1}, \ldots, x_{r+k}\right) d \mu_{r+k}\left(x_{1}, \ldots, x_{r+k}\right) \\
& =\int g(x) \psi \circ f(x) d \mu_{n}(x) \\
& =\int g(x) d \mu_{n}(x) \int \psi \circ f(x) d \mu_{n}(x) \\
& =\int\left(\tilde{g}\left(x_{1}, \ldots, x_{r}\right)+\left\langle\alpha_{2}, \sum_{i=r+1}^{r+k} x_{i} e_{i}\right\rangle\right) d \mu_{r+k}\left(x_{1}, \ldots, x_{r+k}\right) \\
& \quad \times \int \psi\left(f_{1}\right)\left(x_{1}, \ldots, x_{r+k}\right) d \mu_{r+k}\left(x_{1}, \ldots, x_{r+k}\right) .
\end{aligned}
$$

Let consider $Y$ a random vector in $\mathbb{R}^{r+k}$ of law $\mu_{r+k}$ and choose, for $\varepsilon>0, \psi(t)=1_{[-\varepsilon, \varepsilon]}\left(t-f_{1}(0)\right)$. We write $Y=\left(Y_{1}, Y_{2}\right)$ where $Y_{1} \in \mathbb{R}^{r}, Y_{2} \in \mathbb{R}^{k}$. So we have:

$$
E\left(\left(\tilde{g}\left(Y_{1}\right)+\left\langle\alpha_{2}, Y_{2}\right\rangle\right) \frac{1_{\left|f_{1}(Y)-f_{1}(0)\right| \leqslant \varepsilon}}{P\left(\left|f_{1}(Y)-f_{1}(0)\right| \leqslant \varepsilon\right)}\right)=E\left(\tilde{g}\left(Y_{1}\right)+\left\langle\alpha_{2}, Y_{2}\right\rangle\right)=E\left(\tilde{g}\left(Y_{1}\right)\right) .
$$


If there exists $x \neq 0$ such that $f_{1}(x)=f_{1}(0)$, and as $f_{1}$ is convex, we obtain:

$$
\forall t \in[0,1], \quad f_{1}(t x)=f_{1}(0) .
$$

But $f_{1}$ is an analytic function, so:

$$
\forall t \in \mathbb{R}, \quad f_{1}(t x)=f_{1}(0) .
$$

Consequently $x \in \mathcal{E}\left(f_{1}\right)$, which implies $x=0$. We have obtained: $\forall x \neq 0, f_{1}(x)>f_{1}(0)$. Now, we use Lemma 4.3 for $f_{1}$. We denote: $\delta(\varepsilon)=\sup \left\{\|x\|, x \in \mathbb{R}^{r+k}, f_{1}(x)-f_{1}(0) \leqslant \varepsilon\right\}$.

$$
\begin{aligned}
& E\left(\left|\tilde{g}\left(Y_{1}\right)+\left\langle\alpha_{2}, Y_{2}\right\rangle-\tilde{g}(0)\right| \frac{1_{\left|f_{1}(Y)-f_{1}(0)\right| \leqslant \varepsilon}}{P\left(\left|f_{1}(Y)-f_{1}(0)\right| \leqslant \varepsilon\right)}\right) \\
& \quad \leqslant E\left(\left|\tilde{g}\left(Y_{1}\right)+\left\langle\alpha_{2}, Y_{2}\right\rangle-\tilde{g}(0)\right| 1_{\|Y\| \leqslant \delta(\varepsilon)} \frac{1_{\left|f_{1}(Y)-f_{1}(0)\right| \leqslant \varepsilon}}{P\left(\left|f_{1}(Y)-f_{1}(0)\right| \leqslant \varepsilon\right)}\right) .
\end{aligned}
$$

We deduce:

$$
\lim _{\varepsilon \rightarrow 0} E\left(\left(\tilde{g}\left(Y_{1}\right)+\left\langle\alpha_{2}, Y_{2}\right\rangle\right) \frac{1_{\left|f_{1}(Y)-f_{1}(0)\right| \leqslant \varepsilon}}{P\left(\left|f_{1}(Y)-f_{1}(0)\right| \leqslant \varepsilon\right)}\right)=\tilde{g}(0) .
$$

\section{Consequently:}

$$
E\left(\tilde{g}\left(Y_{1}\right)\right)=\tilde{g}(0) .
$$

The map $\tilde{g}$ is convex, that means that there is equality in Jensen's inequality. We deduce $\tilde{g}$ is an affine map, but this is impossible because $\mathcal{E}(\tilde{g})=\{0\}$. Consequently $r=0$. Then, it is possible to find an orthogonal transformation $U$ such that:

$$
f(U x)=\bar{f}\left(x_{r+1}, \ldots, x_{r+k}\right), \quad g(U x)=\bar{g}\left(x_{r+k+1}, \ldots, x_{n}\right)+a x_{r+1} .
$$

But $f(U X)$ and $g(U X)$ are independent random variables, so, $a=0$ or $\bar{f}$ does not depend on $x_{r+1}$ (see the proof of Theorem 4.1).

Remark 11. The idea of using a function like $1_{[-\varepsilon, \varepsilon]}\left(t-f_{1}(0)\right)$ and the case of equality in Jensen's inequality is due to Bhandari and Basu [5]. If we compare the proof given here to their proof, the novelty is the use of $\mathcal{E}(g)$, the choice of the orthonormal basis, the extension to an entire function $f$ and the end of the proof (to prove $a=0$ or $\bar{f}$ does not depend on $x_{r+1}$ ).

Remark 12. In the proof, we could use $\left\{x \in \mathbb{R}^{n}, \forall t \in \mathbb{R}, f(t x)=f(0)\right\}$ instead of $\mathcal{E}(f)$. However, it is essential to work with $\mathcal{E}(g)$. We have to notice that $f(x) \geqslant f(0)$ for all $x$ implies $\left\{x \in \mathbb{R}^{n}, \forall t \in \mathbb{R}, f(t x)=f(0)\right\}=\mathcal{E}(f)$ (by using $\alpha_{1}=0$ and Remark 6).

\section{References}

[1] D. Bakry, L'hypercontractivité et son utilisation en théorie des semi-groupes, in: Ecole d'été de probabilités de Saint-Flour XXII-1992, in: Lecture Notes in Math., vol. 1581, 1994, pp. 1-114.

[2] D. Bakry, Functional inequalities for Markov semigroups, preprint.

[3] D. Bakry, D. Michel, Sur les inégalités F.K.G., in: Séminaire de Probabilités XXVI, in: Lecture Notes in Math., vol. 1526, 1992, pp. 170188.

[4] W. Beckner, A generalized Poincaré inequality for Gaussian measures, Proc. Amer. Math. Soc. 105 (1989) 397-400.

[5] S.K. Bhandari, A. Basu, On the unlinking conjecture of independent polynomial functions, preprint.

[6] S.K. Bhandari, S. DasGupta, Unlinking theorem for symmetric convex functions, in: Multivariate Analysis and its Applications, in: IMS Lecture Notes - Monograph Series, vol. 24, 1994.

[7] G. Hargé, A particular case of correlation inequality for the Gaussian measure, Ann. Probab. 27 (4) (1999) 1939-1951. 
[8] G. Hargé, Inequalities for the Gaussian measure and an application to Wiener space, C. R. Acad. Sci., Série 1 333 (2001) 791-794.

[9] C. Houdré, V. Pérez-Abreu, D. Surgailis, Interpolation, correlation identities, and inequalities for infinely divisible variables, J. Fourier Anal. Appl. 46 (1998) 651-668.

[10] Y. Hu, Itô-Wiener chaos expansion with exact residual and correlation, variance inequalities, J. Theoret. Probab. 10 (1997) 835-848.

[11] A.M. Kagan, Y.V. Linnik, C.R. Rao, Characterization Problems in Mathematical Statistics, Wiley, New York, 1973.

[12] M. Ledoux, L'algèbre de Lie des gradients itérés d'un générateur Markovien - Développements de moyennes et entropies, Ann. Sci. Ecole Norm. Sup. (4) 28 (1995) 435-460.

[13] M. Ledoux, Concentration of measure end logarithmic Sobolev inequalities, in: Séminaire Proba. XXXIII, in: Lecture Notes in Math., vol. 1709, Springer-Verlag, 1999, pp. 120-216.

[14] M. Ledoux, The geometry of Markov diffusion generators, Ann. Fac. Sci. Toulouse 9 (2000) 305-366.

[15] P.A. Meyer, Note sur les processus d'Ornstein-Uhlenbeck, in: Séminaire Proba. XVI, in: Lecture Notes in Math., vol. 920, Springer-Verlag, 1982.

[16] D.L. Ocone, A guide to the stochastic calculus of variations, in: Stochastic Analysis and Related Topics, in: Lecture Notes in Math., vol. 1316, Springer-Verlag, 1986.

[17] L.D. Pitt, A gaussian correlation inequality for symetric convex sets, Ann. Probab. 5 (1977) 470-474. 